\title{
Thermodynamics of a Two-Step Electroweak Phase Transition
}

\author{
Lauri Niemi®, ${ }^{1, *}$ Michael J. Ramsey-Musolf, ${ }^{2,3,4, \dagger}$ Tuomas V. I. Tenkanen, ${ }^{5, \ddagger}$ and David J. Weir $\circledast^{1,6, \S}$ \\ ${ }^{1}$ Department of Physics and Helsinki Institute of Physics, P.O. Box 64, FI-00014 University of Helsinki, Finland \\ ${ }^{2}$ Tsung-Dao Lee Institute and School of Physics and Astronomy, Shanghai Jiao Tong University, \\ 800 Dongchuan Road, Shanghai 200240, China \\ ${ }^{3}$ Amherst Center for Fundamental Interactions, Department of Physics, University of Massachusetts, \\ Amherst, Massachusetts 01003, USA \\ ${ }^{4}$ Kellogg Radiation Laboratory, California Institute of Technology, Pasadena, California 91125, USA \\ ${ }^{5}$ Albert Einstein Center for Fundamental Physics, Institute for Theoretical Physics, University of Bern, \\ Sidlerstrasse 5, CH-3012 Bern, Switzerland \\ ${ }^{6}$ School of Physics and Astronomy, University of Nottingham, Nottingham NG7 2RD, United Kingdom
}

(Received 6 July 2020; accepted 2 March 2021; published 27 April 2021)

\begin{abstract}
New field content beyond that of the standard model of particle physics can alter the thermal history of electroweak symmetry breaking in the early Universe. In particular, the symmetry breaking may have occurred through a sequence of successive phase transitions. We study the thermodynamics of such a scenario in a real triplet extension of the standard model, using nonperturbative lattice simulations. Twostep electroweak phase transition is found to occur in a narrow region of allowed parameter space with the second transition always being first order. The first transition into the phase of nonvanishing triplet vacuum expectation value is first order in a non-negligible portion of the two-step parameter space. A comparison with two-loop perturbative calculation is provided and significant discrepancies with the nonperturbative results are identified.
\end{abstract}

DOI: 10.1103/PhysRevLett.126.171802

Introduction.-In the standard model (SM) of particle physics, electroweak (EW) gauge symmetry is spontaneously broken by the vacuum-expectation value (VEV) of the Higgs field. Thermal corrections to the Higgs potential restore this symmetry in the early Universe. For the physical Higgs mass this transition is a smooth crossover rather than a true phase transition [1-3], i.e., there is no distinction between the symmetric and broken "phases." In many beyond the standard model (BSM) scenarios, the introduction of additional scalar fields can result in a scalar potential having vastly different thermal behavior from that of the SM. In particular, these extensions may yield a bona fide electroweak phase transition (EWPT) that is first order, with cosmological consequences that include conditions needed to generate the cosmic matter-antimatter asymmetry through electroweak baryogenesis (EWBG) [4-6] and production of gravitational waves $(\mathrm{GW})$. A conclusive test of this possibility could result from present and future high energy collider experiments [7] and GW probes [8-10].

An extended scalar potential may admit a richer thermal history than in the SM. The new fields may have phase

Published by the American Physical Society under the terms of the Creative Commons Attribution 4.0 International license. Further distribution of this work must maintain attribution to the author(s) and the published article's title, journal citation, and DOI. Funded by SCOAP ${ }^{3}$. transitions of their own, and the Universe may undergo several symmetry-breaking transitions before settling down to the present EW vacuum. While such a thermal history would be interesting in itself, multistep EW symmetry breaking could have important implications for cosmology. Specifically, EWBG could be realized in a sequence of symmetry-breaking transitions around the EW scale [11-15]. This setup also leads naturally to a strong firstorder transition into the final EW phase through a tree-level potential barrier. Furthermore, a nonminimal pattern of EW symmetry breaking can produce topological solitons, such as monopoles and domain walls, with potentially interesting properties. Such defects are absent in the SM, but are generic in grand unified theories [16]; many analogs also exist in condensed matter systems [17].

The simplest extension of the SM scalar sector admitting distinct phases of broken EW symmetry in the early Universe is the real triplet model with three BSM degrees of freedom, collectively denoted by $\Sigma$. In the resulting " $\Sigma$ SM," EW symmetry breaking may occur directly in a single step from the unbroken phase $O$ to the Higgs phase $\phi$, or in two steps, $O \rightarrow \Sigma \rightarrow \phi$, where EW symmetry is broken in both the $\Sigma$ and $\phi$ phases. A delineation of the model parameters leading to either possibility is given in the perturbative analysis in Ref. [12]. Analogous studies in other models containing new scalars either charged or neutral under the SM gauge symmetries indicate that 
multistep transitions may arise generically [11,14,18-25]. Thus, a more thorough investigation of the thermal history and phase diagram of the $\Sigma \mathrm{SM}$ is well-motivated.

A robust determination of the phase diagram is a nontrivial task even for theories that are weakly coupled at zero temperature. The EWPT is driven by infrared (IR) bosonic fields, the Matsubara zero modes, whose mutual interactions are boosted by Bose enhancement. This results in a poor convergence of perturbation theory and ultimately renders the momentum scale $\sim g^{2} T$ nonperturbative, $g$ being a gauge coupling [26]. This problem affects gauge bosons in the symmetric high-temperature phase and scalar fields near a phase transition where their correlation lengths can grow large. Indeed, perturbation theory incorrectly predicts a first-order EWPT in the minimal SM. There is no a priori reason to trust the perturbative description in BSM settings either, unless one is interested solely in properties of the Higgs phase, where the VEV provides a perturbative mass for most excitations. Large couplings in the scalar sector may further aggravate the IR problem [27,28].

For the EW theory, a solution to the IR problem is known: the thermodynamics are well described by a threedimensional (3D) effective field theory (EFT) for which nonperturbative lattice simulations can be carried out [29-31]. This "dimensional reduction" amounts to perturbatively integrating out nonzero Matsubara modes, and the resulting theory describes thermal fluctuations of the bosonic zero modes.

Here, we report on a nonperturbative study of the $\Sigma$ SM using the $3 \mathrm{~d}$ EFT. The results are used to obtain a realistic picture of the two-step EWPT scenario. We also assess the performance of the perturbative treatment in light of our nonperturbative results.

Model.-The color neutral scalar field $\Sigma$ carries no hypercharge, transforms under the adjoint representation of $\mathrm{SU}(2)_{L}$, and does not couple to SM fermions. For simplicity, we further require invariance under the $Z_{2}$ transformation $\Sigma \rightarrow-\Sigma$, which allows for the VEV $v_{\Sigma}$ to vanish at $T=0$. Doing so ensures consistency with bounds on the EW $\rho$ parameter while enabling the neutral field $\Sigma^{0}$ to contribute to the dark matter relic density $[32,33]$. Recent studies of the corresponding collider and dark matter phenomenology appear in Refs. [34,35]. The most general, renormalizable scalar potential then reads

$$
\begin{aligned}
V(\phi, \Sigma)= & -\mu_{\phi}^{2} \phi^{\dagger} \phi-\frac{1}{2} \mu_{\Sigma}^{2} \Sigma^{a} \Sigma^{a}+\lambda\left(\phi^{\dagger} \phi\right)^{2} \\
& +\frac{b_{4}}{4}\left(\Sigma^{a} \Sigma^{a}\right)^{2}+\frac{a_{2}}{2} \phi^{\dagger} \phi \Sigma^{a} \Sigma^{a},
\end{aligned}
$$

where $a=1,2,3$ is the adjoint index, with $\sqrt{2} \Sigma^{ \pm}=\Sigma^{1} \mp$ $i \Sigma^{2}$ and $\Sigma^{0}=\Sigma^{3}$.

For $\mu_{\phi}^{2}>0$, the potential has a symmetry-breaking minimum in the Higgs direction, $\left\langle\phi^{\dagger} \phi\right\rangle=\frac{1}{2} v^{2}$ with $v_{\Sigma}=0$. This corresponds to the standard EW minimum with three BSM excitations from the $\Sigma$ field, whose masses are degenerate at tree level [32]. Following Ref. [36], we relate the Lagrangian parameters to EW observables through pole-mass renormalization at the one-loop level, taking the mass $M_{\Sigma}$ of $\Sigma^{0}$ as an input parameter. We treat the couplings $a_{2}$ and $b_{4}$ as input parameters directly at the $\overline{\mathrm{MS}}$ scale $M_{Z}$. The one-loop correction is necessary to match the accuracy of our EFT construction below.

If $\mu_{\Sigma}^{2}>0$, a second minimum of $V(\phi, \Sigma)$ appears in the $\Sigma$ direction, with $v=0$. In this $\Sigma$ vacuum phase, the system admits't Hooft-Polyakov monopole excitations [37-40].

Thermal corrections can modify the vacuum structure. In the high- $T$ limit, the leading effect is a $T$-dependent reduction of the squared mass parameters: $\mu_{\phi, \Sigma}^{2} \rightarrow \mu_{\phi, \Sigma}^{2}-$ $\Pi_{\phi, \Sigma} T^{2}$. Here $\Pi_{\phi, \Sigma}$ are $\mathcal{O}\left(g^{2}\right)$ constants, where $g^{2}$ denotes a general quartic coupling. The thermal correction turns $\mu_{\phi}^{2}$ negative at $T_{\phi} \sim 100 \mathrm{GeV}$, relaxing the Higgs VEV to zero. Two-step EWSB occurs if the thermal corrections drive $\mu_{\Sigma}^{2}$ negative at a higher temperature $T_{\Sigma}>T_{\phi}$. The Universe then resides in the symmetric phase $O$ at high temperatures before transitioning into the $\Sigma$ phase $(O \rightarrow \Sigma)$ at $T_{\Sigma}$, followed by another phase transition $(\Sigma \rightarrow \phi)$ into the final Higgs phase at $T_{\phi}$. The presence of a tree-level saddle point separating the $\phi$ and $\Sigma$ minima suggests a firstorder transition in the second stage.

High-T EFT.-We derive the 3D EFT in the imaginary time formalism by integrating out modes with a nonzero Matsubara frequency, including all fermions, leading to the Euclidean space Lagrangian:

$$
\begin{aligned}
\mathcal{L}_{3 \mathrm{~d}}= & \frac{1}{4}\left(F_{i j}^{a}\right)^{2}+\left|D_{i} \phi\right|^{2}+\frac{1}{2}\left(D_{i} \Sigma^{a}\right)^{2}+\bar{\mu}_{\phi}^{2} \phi^{\dagger} \phi+\bar{\lambda}\left(\phi^{\dagger} \phi\right)^{2} \\
& +\frac{\bar{\mu}_{\Sigma}^{2}}{2} \Sigma^{a} \Sigma^{a}+\frac{\bar{b}_{4}}{4}\left(\Sigma^{a} \Sigma^{a}\right)^{2}+\frac{\bar{a}_{2}}{2} \phi^{\dagger} \phi \Sigma^{a} \Sigma^{a} .
\end{aligned}
$$

Here, $F_{i j}^{a}$ is the $\mathrm{SU}(2)_{L}$ field strength tensor. Thermal corrections from the hard scale $\pi T$ are included in the barred parameters, whose matching was worked out to $\mathcal{O}\left(g^{4}\right)$ accuracy in Ref. [36] and includes corrections from temporal components of the gauge fields that generate a Debye screening mass and can be integrated out $[31,36]$. These couplings in Eq. (2) are dimensionful, and the fields are scaled by $T^{-1 / 2}$. We have neglected the $\mathrm{U}(1)_{Y}$ gauge field and the $\mathrm{SU}(3)_{C}$ sector as they have only a small effect on the EWPT [41] and do not couple to $\Sigma$.

The EFT is formally valid in the high- $T$ limit $m \ll \pi T$. By construction, its region of validity overlaps with that of the consistent daisy resummation of Ref. [42] as required to correctly describe physics at the "soft" scale $g T$. The EFT systematically includes these corrections.

To probe the parameter space for a two-step EWPT, we have scanned the parameters using the effective potential $V_{\text {eff }}$ calculated to two-loop order in the EFT. Evolution of the different minima is tracked using the gauge-invariant 


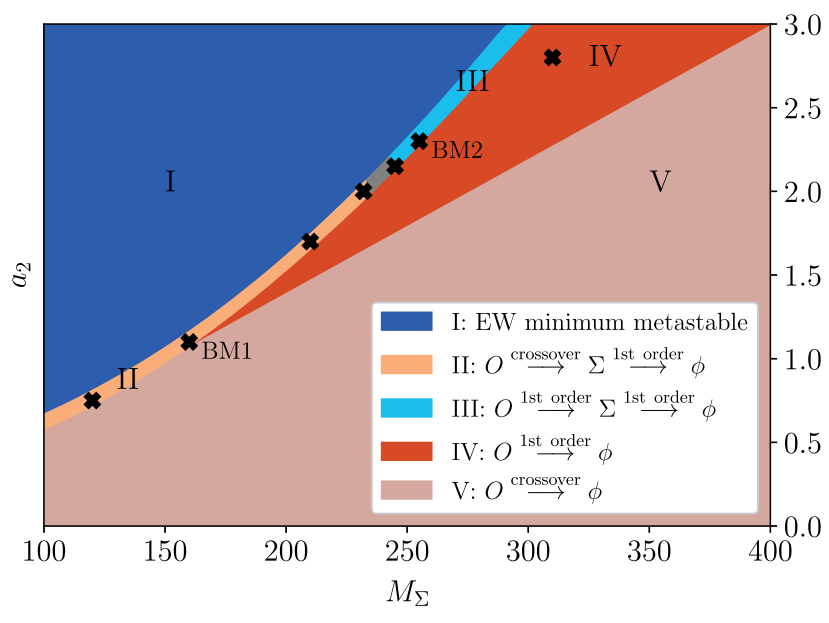

FIG. 1. $\Sigma$ SM phase diagram for $\Sigma$ self-coupling parameter $b_{4}=0.25$. Vertical and horizontal axes give the triplet-Higgs coupling and triplet mass, respectively. Colored regions correspond to different types of EW symmetry-breaking transitions: direct transitions into the Higgs phase (regions IV and V); twostep symmetry breaking with different types of the $O \rightarrow \Sigma$ transition (II for crossover, III for first order, gray uncertain); unstable EW minimum at $T=0$ (I). Both $O \rightarrow \phi$ and $O \rightarrow \Sigma$ transitions grow stronger as the quartic portal coupling $a_{2}$ increases. Crosses indicate lattice benchmarks; see text for a detailed discussion of BM1 and BM2.

approach described in Refs. [43,44]; details appear in the Supplemental Material [45]. Two-step transitions occur in a narrow band separating the parameter space of one-step EWPTs $(O \rightarrow \phi)$ from that where the EW minimum is metastable at $T=0$. For $b_{4}=0.25$, this is illustrated in Fig. 1.

In much of parameter space, the EWPT is driven solely by the Higgs doublet, which becomes parametrically light near the critical temperature, $\bar{\mu}_{\phi}^{2} \sim\left(g^{2} T\right)^{2}$, due to a cancellation between vacuum and thermal masses. This allows us to integrate out $\Sigma$ as an UV mode near the critical temperature $T_{c}$, resulting in a simpler EFT for which the nonperturbative phase diagram is known $[1,50]$. This approach was taken in Ref. [36] to identify where the $O \rightarrow \phi$ transition is a crossover: region V of Fig. 1 . Deep in region IV, integrating out $\Sigma$ is no longer justified, but our simulations verify that the transition remains first order here. The line separating regions IV and V corresponds to second order transitions. Its location is only accurate within $\sim 10 \%$ due to neglect of higher-dimensional operators [36].

It is interesting to ask where in the two-step EWPT region the $O \rightarrow \Sigma$ transition is first order. As in the SM case, perturbation theory does not provide reliable guidance; genuine nonperturbative input is needed. Qualitatively, at temperatures near a $O \rightarrow \Sigma$ transition we expect the IR physics to resemble that of a GeorgiGlashow type theory containing just gauge fields and $\Sigma$ [51]. The corresponding phase transition terminates at a finite value of the scalar self coupling [52]. Our simulations confirm this expectation: the $O \rightarrow \Sigma$ transition is crossover in region II; first order in region III; and terminates somewhere in the gray region between. We have not attempted a more precise determination of the end line.

The IR behavior also suggests that the $O \rightarrow \Sigma$ transition grows stronger at small $b_{4}$, which we have verified with simulations using $b_{4}=0.15,0.20$. However, the two-step region itself becomes narrower due to a decrease in the $\Sigma$ minimum vacuum energy, $\sim-\mu_{\Sigma}^{4} /\left(4 b_{4}\right)$ at tree level. There is no two-step EWPT if the $T=0$ potential is deeper in the $\Sigma$ direction than in the Higgs minimum (region I).

Simulations.-Simulations in the full $\Sigma$ SM are not practical due to the chirally coupled fermions. A systematic method for implementing the fermionic corrections (which are significant) is provided by the dimensionally reduced EFT (2). To discretize it, we employ the (unimproved) Wilson action for the gauge links and couple these to the scalars through gauge-invariant hopping terms. Parameters in the lattice action are related to the continuum parameters in Eq. (2) by expressions given in Ref. [53]. These relations become exact in the continuum limit as a consequence of superrenormalizability of the 3D EFT.

In lattice simulations, we determine probability distributions of gauge-invariant operators by generating field configurations in the canonical ensemble. For the EWPT, the observables of interest are scalar condensates, particularly $\left\langle\phi^{\dagger} \phi\right\rangle$ and $\left\langle\Sigma^{a} \Sigma^{a}\right\rangle$, whose probability distributions in a first order transition develop a two-peak structure. The peaks correspond to the bulk phases and have equal integrated probabilities at $T_{c}$ [50].

In the region separating the bulk phases, the ensemble is dominated by mixed-phase configurations where the two phases exist simultaneously on the lattice [54]. The phase interface carries free energy proportional to its surface area, and the probability of tunneling between phases is thus exponentially suppressed. This makes it difficult to obtain the probability distributions using conventional update algorithms for the canonical ensemble. To enhance the phase transition signal, we apply multicanonical simulations [55], where the ensemble is modified by a suitable weight function $W$ as $\exp [-S] \rightarrow \exp \left[-S-W\left(\Phi_{\text {multi }}\right)\right]$, where $\Phi_{\text {multi }}$ is typically an order parameterlike quantity that distinguishes the phases. The canonical distributions are then obtained by reweighting the measurements [56]. While $W$ itself can be calculated recursively [57,58], the efficiency of multicanonical simulations depends on the choice of $\Phi_{\text {multi }}$. For $O \rightarrow \Sigma$ transitions, we choose the volume average of $\Sigma^{a} \Sigma^{a}$, and the simulations proceed analogously to those of Refs. [50,52].

Consistent with perturbation theory we found that, for all the cases we studied (crosses in Fig. 1), the $\Sigma \rightarrow \phi$ stage is a first-order transition with strong suppression of the mixed configurations. We have not found a simple choice of $\Phi_{\text {multi }}$ that would efficiently take the system both ways between 


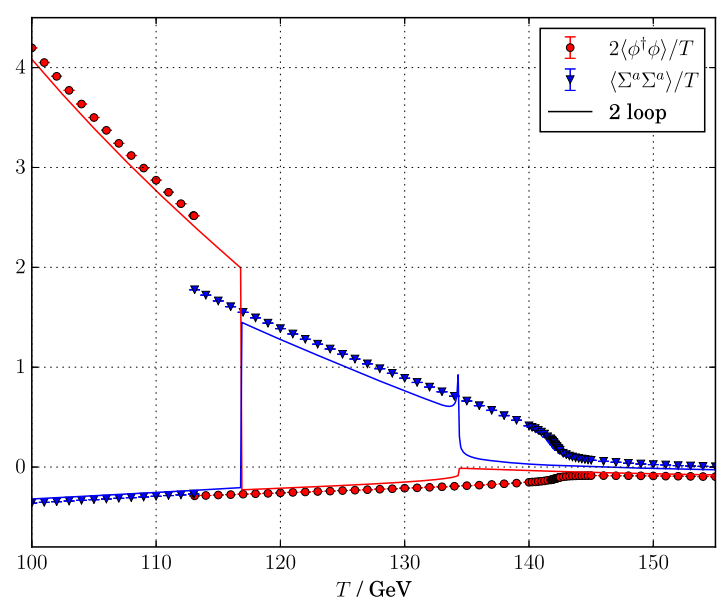

(a) BM1: $\left(M_{\Sigma}, a_{2}, b_{4}\right)=(160 \mathrm{GeV}, 1.1,0.25)$

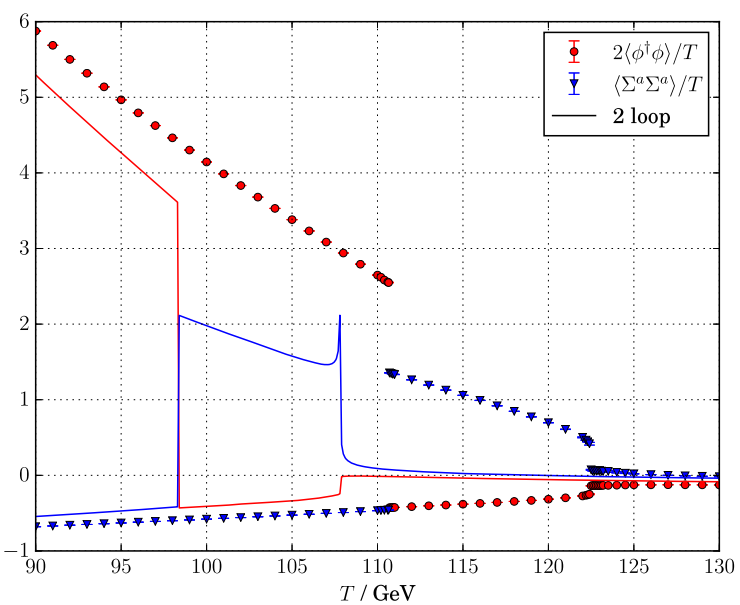

(b) BM2: $\left(M_{\Sigma}, a_{2}, b_{4}\right)=(255 \mathrm{GeV}, 2.3,0.25)$

FIG. 2. Temperature dependence of quadratic condensates in the 3D EFT as measured on the lattice in the global probability maximum and converted to the $\overline{\mathrm{MS}}$ scheme (at scale $T$ ) using relations in Ref. [53]. The solid lines are perturbative estimates at two loop order in the same EFT. Results shown are for a $60^{3}$ lattice with $4 /\left(a \bar{g}^{2}\right)=24$, large enough for finite-size effects to be negligible. Monte Carlo statistical errors are too small to be visible at this scale.

the two broken phases. In either phase, one of the scalar condensates develops large bulk fluctuations, and an even larger fluctuation is required to start the tunneling process. Instead, we determine $T_{c}$ by restricting the simulation to sample the mixed-phase configurations only. At $T_{c}$, neither phase is preferred over the other, and probability distributions of order parameters in the allowed range become approximately flat.

The simulation results carry mild dependence on lattice volume and spacing $a$, but extrapolations $V \rightarrow \infty$ and $a \rightarrow 0$ can be taken in a controlled fashion [50,59,60]. For the first-order transitions studied here, $\mathcal{O}(a)$ errors appear negligible for $4 /\left(a \bar{g}^{2}\right) \geq 20$, with both $T_{c}$ and condensate values changing by less than $1 \%$ if $a$ is decreased. Volume dependence appears to be even smaller, suggesting that our finite-size effects are well under control. Below we quote results from only the largest lattices.

Our code for simulating the $\mathrm{SU}(2)$ theory with fundamental and adjoint Higgs's has been cross-checked by reproducing histograms in Refs. [50,52]. We employ conventional heat bath updates for the gauge links [61] and a mixture of Metropolis and overrelaxation updates [50] for the scalars.

Results and discussion.-The condensates require additive renormalization, but their discontinuities across a phase transition are renormalization group invariant and directly related to the latent heat $L[53,62]$, a physical quantity characterizing transition strength. Figure 2 shows the condensate evolution for two benchmark (BM) points giving a two-step EWPT, together with perturbative estimates. In the high- $T$ phase the condensates stay close to zero, while at low temperatures $\left\langle\phi^{\dagger} \phi\right\rangle$ obtains a large value. The existence of an intermediate $\Sigma$ phase is clearly visible.
The condensates can be negative because of the additive renormalization.

In BM1, the $O \rightarrow \Sigma$ stage is a crossover: we find no evidence of phase coexistence, ruling out a first-order transition. To investigate the possibility of a second order transition we studied finite-size scaling of the dimensionless $\Sigma^{2}$ susceptibility,

$$
\chi\left(\Sigma^{2}\right)=\frac{1}{4} V T\left[\left\langle\left(\Sigma^{a} \Sigma^{a}\right)_{V}^{2}\right\rangle-\left\langle\left(\Sigma^{a} \Sigma^{a}\right)_{V}\right\rangle^{2}\right]
$$

where the subscript denotes volume averaging. As shown in Fig. 3, $\chi\left(\Sigma^{2}\right)$ peaks at $T \approx 142 \mathrm{GeV}$ but converges to a finite value as $V \rightarrow \infty$, consistent with crossover behavior.

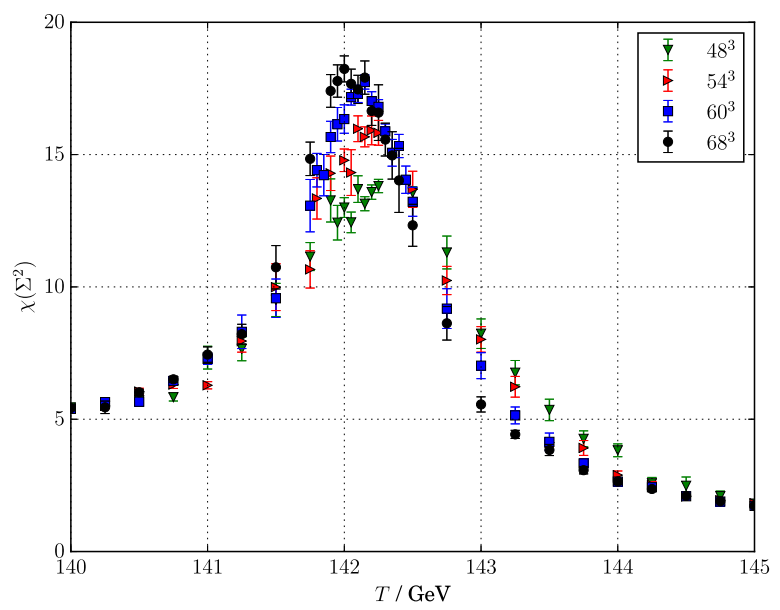

FIG. 3. $T$ dependence of $\Sigma^{a} \Sigma^{a}$ susceptibility (3) across the crossover in BM1, measured at $4 /\left(a \bar{g}^{2}\right)=24$ and different volumes. 
By contrast, for a second-order transition the susceptibility diverges with a critical exponent. The first transition in BM2 is first order and, depending on the criterion for baryon number preservation within the $\Sigma$ phase, could be strong enough to support two-step EWBG [12].

To assess the reliability of perturbation theory, we compare the nonperturbative results to those obtained from the two-loop $V_{\text {eff }}$ (solid lines in Fig. 2). In the gaugeinvariant treatment used here, the potential is minimized by expanding the VEVs around their tree-level values (see the Supplemental Material [45]). Near the $O \rightarrow \Sigma$ transition, this approach breaks down due to the absence of a small expansion parameter, and the potential encounters an IR divergence $[43,62]$. This is the reason for the spiking of $\left\langle\Sigma^{a} \Sigma^{a}\right\rangle / T$ in Fig. 2. Consequently, the crossover in BM1 is not visible perturbatively.

Outside the temperature range of $O \rightarrow \Sigma$ transitions, perturbation theory provides some rough qualitative guidance but performs poorly in quantitatively describing both $T_{c}$ and the "strength" (condensate discontinuities). For the $\Sigma \rightarrow \phi$ transition this finding is, perhaps, surprising, as the two minima are present already in the tree-level potential. Nevertheless, scalar loops can significantly alter the transition dynamics because of the large $a_{2}$ coupling necessary for a two-step EWPT. On dimensional grounds, the high- $T$ expansion parameter for $\phi-\Sigma$ interactions is of the form $a_{2} T \times(\text { scalar mass })^{-1}$. At the second transition, the masses are bounded from below by the nonzero VEVs but are numerically small compared to $a_{2} T$. Hence, a low-order perturbative description is not necessarily reliable. There may also be additional nonperturbative effects from magnetic monopoles that exist in the $\Sigma$ phase.

After extrapolating $V \rightarrow \infty$ and $a \rightarrow 0$, the latent heat is $L / T_{c}^{4}=0.4109(2)$ for the $\Sigma \rightarrow \phi$ transition in BM1; in BM2 the value for the first (second) transition is 0.151(2) [0.5895(9)]. Errors are the stastistical uncertainties. The perturbative values, where applicable, are smaller by $30 \%$ in BM1 and larger by $40 \%$ in BM2. The discrepancy is dominated by the error in $T_{c}$. The two-loop potential is crucial for even a qualitative agreement with the nonperturbative results: at one loop, the jumps in condensates are more than 50\% smaller than at two loops, while the temperatures differ only by a few percent.

Applicability of these results to the full 4D $\Sigma$ SM depends on the overall accuracy of our 3D EFT. Dimensional reduction produces operators of dimension six (in 4D units) that we have neglected here. We anticipate that the operators $c_{\phi}\left(\phi^{\dagger} \phi\right)^{3} / T^{2}$ and $c_{\Sigma}\left(\Sigma^{a} \Sigma^{a}\right)^{3} / T^{2}$ yield the largest contribution, with a potentially significant effect in the presence of a nonvanishing condensate. Following Ref. [31], we estimate their effects on scalar VEVs at tree level. For $T>50 \mathrm{GeV}$, the operators cause relative shifts of less than $1 \%$ in the VEVs in both BM1 and BM2, suggesting that the performance of our dimensional reduction is comparable to the SM case, despite the relatively heavy scalar excitations in the Higgs phase.

Overall, our results for the $\Sigma \mathrm{SM}$ phase diagram (Fig. 1) validate the expectations from purely perturbative studies that the early universe could have undergone successive EWSB transitions. To our knowledge, this work provides the first nonperturbative demonstration of this possiblility. Indeed, a robust determination of the character of these transitions and a quantitative determination of their properties $\left(T_{C}\right.$, latent heat, and model parameter dependence) requires a nonperturbative treatment. We anticipate that future nonperturbative studies will be essential for obtaining dynamical properties (e.g., rates for nucleation [54], sphaleron transitions [63], and monopole-catalyzed processes [64]) necessary for a complete picture of the associated thermal history in the $\Sigma \mathrm{SM}$ and other extended scalar sector scenarios. In this context, we consider the present study as the first step in an exciting program aimed at building a rigorous understanding of nonminimal electroweak symmetry breaking.

We thank Oliver Gould, Mark Hindmarsh, Kimmo Kainulainen, Mikko Laine, Arttu Rajantie and Kari Rummukainen for discussions, as well as Philipp Schicho and Juuso Österman for useful correspondence on 3D loop integrals. L. N. acknowledges financial support from the Jenny and Antti Wihuri Foundation. This work was partly supported by the Swiss National Science Foundation (SNF) under Grant No. 200020B-188712, and by the Academy of Finland under Grants 308791 and 320123. M. J. R. M. was supported in part under U.S. Department of Energy Contract No. DE-SC0011095 and National Science Foundation of China Grant No. 19Z103010239. We are grateful for computational resources provided by the University of Helsinki clusters (urn:nbn:fi:research-infras-2016072533). D. J. W. (ORCID ID 0000-0001-6986-0517) was supported by a Science and Technology Facilities Council Ernest Rutherford Fellowship, Grant No. ST/R003904/1, by the Academy of Finland, Grants No. 324882 and No. 328958, and by the Research Funds of the University of Helsinki.

*lauri.b.niemi@helsinki.fi

†mjrm@sjtu.edu.cn,mjrm@physics.umass.edu tenkanen@itp.unibe.ch

§avid.weir@helsinki.fi

[1] K. Kajantie, M. Laine, K. Rummukainen, and M. E. Shaposhnikov, Phys. Rev. Lett. 77, 2887 (1996).

[2] F. Csikor, Z. Fodor, and J. Heitger, Phys. Rev. Lett. 82, 21 (1999).

[3] M. D'Onofrio and K. Rummukainen, Phys. Rev. D 93, 025003 (2016).

[4] V. A. Kuzmin, V. A. Rubakov, and M. E. Shaposhnikov, Phys. Lett. 155B, 36 (1985).

[5] M. E. Shaposhnikov, Pis'ma Zh. Eksp. Teor. Fiz. 44, 364 (1986) [JETP Lett. 44, 465 (1986)]. 
[6] M. E. Shaposhnikov, Nucl. Phys. B287, 757 (1987).

[7] M. J. Ramsey-Musolf, J. High Energy Phys. 09 (2020) 179.

[8] C. Caprini et al., J. Cosmol. Astropart. Phys. 04 (2016) 001.

[9] F. P. Huang, Y. Wan, D.-G. Wang, Y.-F. Cai, and X. Zhang, Phys. Rev. D 94, 041702(R) (2016).

[10] C. Caprini et al., J. Cosmol. Astropart. Phys. 03 (2020) 024.

[11] A. Hammerschmitt, J. Kripfganz, and M. Schmidt, Z. Phys. C 64, 105 (1994).

[12] H. H. Patel and M. J. Ramsey-Musolf, Phys. Rev. D 88, 035013 (2013).

[13] S. Inoue, G. Ovanesyan, and M. J. Ramsey-Musolf, Phys. Rev. D 93, 015013 (2016).

[14] N. Blinov, J. Kozaczuk, D. E. Morrissey, and C. Tamarit, Phys. Rev. D 92, 035012 (2015).

[15] M. J. Ramsey-Musolf, P. Winslow, and G. White, Phys. Rev. D 97, 123509 (2018).

[16] R. Jeannerot, J. Rocher, and M. Sakellariadou, Phys. Rev. D 68, 103514 (2003).

[17] W. Zurek, Phys. Rep. 276, 177 (1996).

[18] S. Profumo, M. J. Ramsey-Musolf, and G. Shaughnessy, J. High Energy Phys. 08 (2007) 010.

[19] J. R. Espinosa, T. Konstandin, and F. Riva, Nucl. Phys. B854, 592 (2012).

[20] H. H. Patel, M. J. Ramsey-Musolf, and M. B. Wise, Phys. Rev. D 88, 015003 (2013).

[21] D. Curtin, P. Meade, and C.-T. Yu, J. High Energy Phys. 11 (2014) 127.

[22] M. Jiang, L. Bian, W. Huang, and J. Shu, Phys. Rev. D 93, 065032 (2016).

[23] G. Kurup and M. Perelstein, Phys. Rev. D 96, 015036 (2017).

[24] C.-W. Chiang, M. J. Ramsey-Musolf, and E. Senaha, Phys. Rev. D 97, 015005 (2018).

[25] Z. Kang, P. Ko, and T. Matsui, J. High Energy Phys. 02 (2018) 115.

[26] A. D. Linde, Phys. Lett. 96B, 289 (1980).

[27] M. Laine, M. Meyer, and G. Nardini, Nucl. Phys. B920, 565 (2017).

[28] K. Kainulainen, V. Keus, L. Niemi, K. Rummukainen, T. V. I. Tenkanen, and V. Vaskonen, J. High Energy Phys. 06 (2019) 075.

[29] P. H. Ginsparg, Nucl. Phys. B170, 388 (1980).

[30] T. Appelquist and R. D. Pisarski, Phys. Rev. D 23, 2305 (1981).

[31] K. Kajantie, M. Laine, K. Rummukainen, and M.E. Shaposhnikov, Nucl. Phys. B458, 90 (1996).

[32] P. Fileviez Perez, H. H. Patel, M. J. Ramsey-Musolf, and K. Wang, Phys. Rev. D 79, 055024 (2009).

[33] M. Cirelli, N. Fornengo, and A. Strumia, Nucl. Phys. B753, 178 (2006).

[34] N. F. Bell, M. J. Dolan, L. S. Friedrich, M. J. Ramsey-Musolf, and R. R. Volkas, J. High Energy Phys. 05 (2020) 050.
[35] C.-W. Chiang, G. Cottin, Y. Du, K. Fuyuto, and M. J. Ramsey-Musolf, J. High Energy Phys. 01 (2021) 198.

[36] L. Niemi, H. H. Patel, M. J. Ramsey-Musolf, T. V. I. Tenkanen, and D. J. Weir, Phys. Rev. D 100, 035002 (2019).

[37] Y. M. Shnir, Magnetic Monopoles, Text and Monographs in Physics (Springer, Berlin/Heidelberg, 2005).

[38] G. 't Hooft, Nucl. Phys. B79, 276 (1974).

[39] A. M. Polyakov, JETP Lett. 20, 194 (1974).

[40] A. Rajantie, Phys. Rev. D 68, 021301(R) (2003).

[41] K. Kajantie, M. Laine, K. Rummukainen, and M. E. Shaposhnikov, Nucl. Phys. B493, 413 (1997).

[42] P. B. Arnold and O. Espinosa, Phys. Rev. D 47, 3546 (1993); [50, 6662(E) (1994).

[43] M. Laine, Phys. Rev. D 51, 4525 (1995).

[44] H. H. Patel and M. J. Ramsey-Musolf, J. High Energy Phys. 07 (2011) 029.

[45] See Supplemental Material at http://link.aps.org/supplemental/ 10.1103/PhysRevLett.126.171802 for a detailed calculation of the two-loop effective potential in the 3D EFT, which includes Refs. [46-49].

[46] K. Farakos, K. Kajantie, K. Rummukainen, and M. E. Shaposhnikov, Nucl. Phys. B425, 67 (1994).

[47] K. G. Chetyrkin and F. V. Tkachov, Nucl. Phys. B192, 159 (1981).

[48] S. Laporta, Int. J. Mod. Phys. A 15, 5087 (2000).

[49] M. Nishimura and Y. Schroder, J. High Energy Phys. 09 (2012) 051.

[50] K. Kajantie, M. Laine, K. Rummukainen, and M. E. Shaposhnikov, Nucl. Phys. B466, 189 (1996).

[51] H. Georgi and S. L. Glashow, Phys. Rev. Lett. 28, 1494 (1972).

[52] K. Kajantie, M. Laine, K. Rummukainen, and M.E. Shaposhnikov, Nucl. Phys. B503, 357 (1997).

[53] M. Laine, Nucl. Phys. B451, 484 (1995).

[54] G. D. Moore and K. Rummukainen, Phys. Rev. D 63, 045002 (2001).

[55] B. A. Berg and T. Neuhaus, Phys. Lett. B 267, 249 (1991).

[56] A. M. Ferrenberg and R. H. Swendsen, Phys. Rev. Lett. 61, 2635 (1988).

[57] M. Laine and K. Rummukainen, Nucl. Phys. B535, 423 (1998).

[58] F. Wang and D. P. Landau, Phys. Rev. Lett. 86, 2050 (2001).

[59] M. Laine and K. Rummukainen, Nucl. Phys. B597, 23 (2001).

[60] M. Laine, G. Nardini, and K. Rummukainen, J. Cosmol. Astropart. Phys. 01 (2013) 011.

[61] A. D. Kennedy and B. J. Pendleton, Phys. Lett. 156B, 393 (1985).

[62] K. Farakos, K. Kajantie, K. Rummukainen, and M. E. Shaposhnikov, Nucl. Phys. B442, 317 (1995).

[63] M. D’Onofrio, K. Rummukainen, and A. Tranberg, Phys. Rev. Lett. 113, 141602 (2014).

[64] V. Rubakov, JETP Lett. 33, 644 (1981). 